\title{
A Soft Robot for Random Exploration of Terrestrial Environments
}

\author{
S. Mintchev, Member, IEEE, D. Zappetti, Student, IEEE, J. Willemin and D. Floreano, Senior \\ Member, IEEE
}

\begin{abstract}
A swarm of randomly moving miniature robots is an effective solution for the exploration of unknown terrains. However, the deployment of a swarm of miniature robots poses two challenges: finding an adequate locomotion strategy for fast exploration and obstacles negotiation; and implementing simple design and control solutions suited for mass manufacturing. Here, we tackle these challenges by developing a new soft robot with a minimalistic design and a simple control strategy that can randomly propel itself above obstacles and roll on the ground upon landing. The robot is equipped with two propellers that are periodically activated to jump, a soft cage that protects the robot from impacts and allows to passively roll on the ground, and a passive self-righting mechanism for repetitive jumps. The minimalistic control and design reduce the complexity of the mechanics and electronics and are instrumental to the production of a large number of robots. In the paper, the key design aspects of the robot are discussed, the locomotion of a single prototype is experimentally characterized, and improvements of the system for future swarm operations are discussed.
\end{abstract}

\section{INTRODUCTION}

In recent years, the use of robots to explore and collect data in unstructured environments has become a relevant research topic with potential applications in the fields of search and rescue [1], space exploration [2][3], environment monitoring [4], and agriculture [5]. Indeed, robots can reach areas that are too dangerous, or even inaccessible to humans, and provide valuable information to their users. In missions that require in situ monitoring, and data collection, terrestrial mobile robots have been preferred over aerial robots that would otherwise need to periodically land and take-off, a challenging task in uneven or cluttered terrains. To date, exploration of terrestrial environments using robots have been performed through either a systematic or a random exploration strategy [6]. Usually, as the information regarding positional or behavioral characteristics of targets decreases, a systematic search become less efficient and more time consuming. In these scenarios, random search strategies are preferred, because they allow to increase the chances to reach and exploring different regions, therefore maximizing the possibility of gathering data [6]. Additionally, because random exploration does not require complex and time-consuming planning and mapping algorithms, a random exploration can be performed with very simple and fast robots. This type of robot is ideal for mass production and swarm operations. A swarm composed of several fast agents can rapidly spread in the environment and achieve high rates of exploration. Furthermore, swarm exploration is robust against failure or malfunctioning of single agents [2].

The authors are with the Laboratory of Intelligent Systems (http://lis.epfl.ch) at Ecole Polytechnique Fédérale de Lausanne (EPFL), CH1015 Lausanne, Switzerland (email: stefano.mintchev@epfl.ch).
Bristelbot [7] and Fiberbot [8] are two examples of simple and fast robot for the random exploration of flat surfaces [7]. These robots exploit a resonant dynamic locomotion to randomly wander on smooth surfaces. They are equipped with a vibration motor and with fibrillar structures with anisotropic frictional properties that convert vibrations into a net forward motion [8]. With this simple locomotion strategy, the robot moves forward until it hits an obstacle, then randomly changes its heading, thus achieving a random exploration of the environment. However, such a locomotion strategy is limited to relatively smooth surfaces because the fibrillary structures are impaired in rough, uneven terrains.

The locomotion of small terrestrial robots in unstructured terrains is often cited as a key research challenge in robotics [9]. Indeed, according to the size-grain hypothesis, the environment becomes more uneven and rugose with the decreasing of robot's size [10]. A common approach to tackle this challenge is to allow small robots to jump above obstacles and subsequently roll upon landing to cover large distances [2]. For over three decades, researchers have developed different types of jumping robots. However, the large majority of jumping robots with on-board energy source exploit complex propulsion and steering mechanisms composed of springs, levers and gears [9]-[14], that can be a limiting factor for the mass production required for swarm applications. A solution to this problem is found in soft jumping robots with a minimalistic mechanical design and an easy to manufacture jumping mechanism [15]-[17]. Another advantage of soft jumping robots is their intrinsic resilience that enhances survivability during landing.

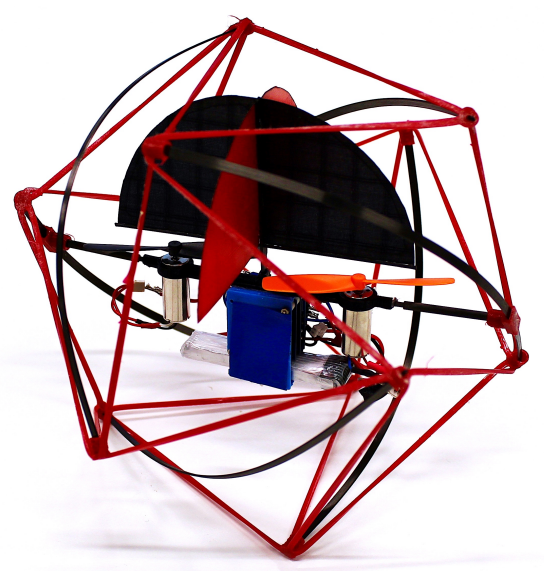

Figure 1. Prototype of the random explorer. The prototype is self-contained being equipped with two propellers that generate the thrust required for jumping, control electronics and a battery for up to 15 minutes of operation. 
In the paper, we propose a novel soft tensegrity robot for the random exploration of uneven terrains. The robot, depicted in Fig. 1, is composed of a soft tensegrity cage that envelops an internal core with a propelled-based propulsion system, control electronics and a battery. The propellers produce the thrust that is required for jumping up to $400 \mathrm{~cm}$ without the need of complex mechanisms. The soft tensegrity cage protects the robot during the landing and allows the robot to roll on the ground in order to increase the distance covered after each jump. A passive, gravity based self-righting mechanism reorients upward the propellers for the subsequent jumps. The main advantages of the proposed robot are its minimalistic design and control strategy, ease of manufacturing, and a dynamic locomotion gait that exploits jumping and passive rolling to rapidly search terrestrial environments.

First, the design and manufacturing of the robot are presented. Secondly, the control strategy that allows to randomly explore is discussed. Thirdly, a prototype of random explorer is experimentally characterized. A discussion of the results and proposals for future work conclude the paper.

\section{Mechanical Design}

The development of a random explorer for exploring uneven and unstructured terrains requires tackling two main design challenges. First, the implementation of an actuation mechanism for jumping locomotion, coupled with a body able to withstand mechanical shocks after jumping and to rapidly roll on the ground. Secondly, a jumping robot requires an uprighting strategy in order to perform repetitive jumps [10]. Other desirable features are a minimalistic mechanical design and control to reduce the complexity of the electronics and of the mechanisms required for functioning. Such features would simplify the fabrication and facilitate the robot production and multi-agent deployment.

All the requirements are fulfilled by the random explorer depicted in Fig. 1. The proposed implementation is based on two elements that work in synergy, a central core and a protective cage (Fig 2). The central core comprises two contrarotating propellers as a propulsion mechanism for jumping and rolling, electronics, battery and an aerodynamic damper to passively stabilize the jump [18] (Fig. 2A). The protective cage has an overall approximated spherical shape to facilitate roll on the ground, and to withstand mechanical shocks upon landing in order to protect the components of the internal core (Fig. 2B).

Compared to the complex jumping mechanisms commonly exploited in state-of-the-art jumping robots [9][10][12], the use of propellers for propulsions allows to drastically simplify the design and manufacturing of the jumping mechanism. The solution with two contra-rotating propellers provides stabilization around the yaw axis during jumping. The simultaneous activation of the two propellers at similar velocities minimizes the destabilization torque generated on the robot preventing the undesired spin around the yaw axis. Stabilization in pitch and roll is provided by an aerodynamic damper [18] (Fig. 2A). It is composed of two perpendicular fins attached to the central core (Fig. 1). The contra-rotating propellers and the aerodynamic damper allow the robot to take-off and jump without the need of attitude sensors and control loops for active stabilization. The damper and the contra-rotating propellers provide just enough stability during the take-off to allow the robot to jump. Afterward, the robot destabilizes and fall on the ground. This instability-driven behavior contributes to the robot's random directions and jumping heights during locomotion.

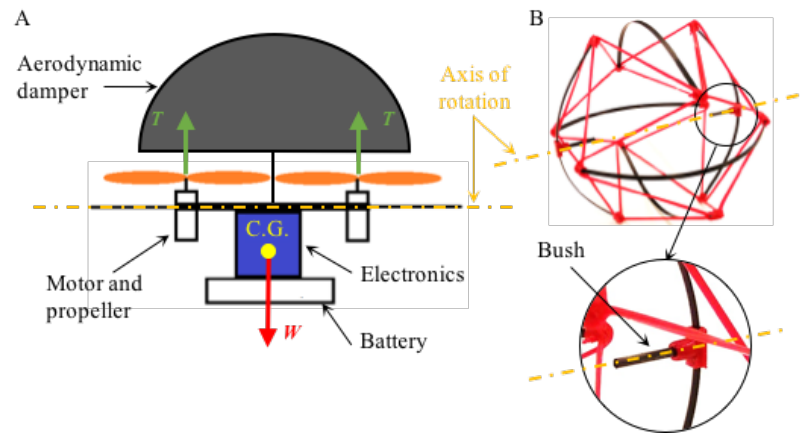

Figure 2. (A) Core of the robot with the main components. The axis of rotation is highlighted by the yellow dashed line. (B) Protective tensegrity cage with detailed view of the right bush where the rotation axis of the core is inserted during assembly.

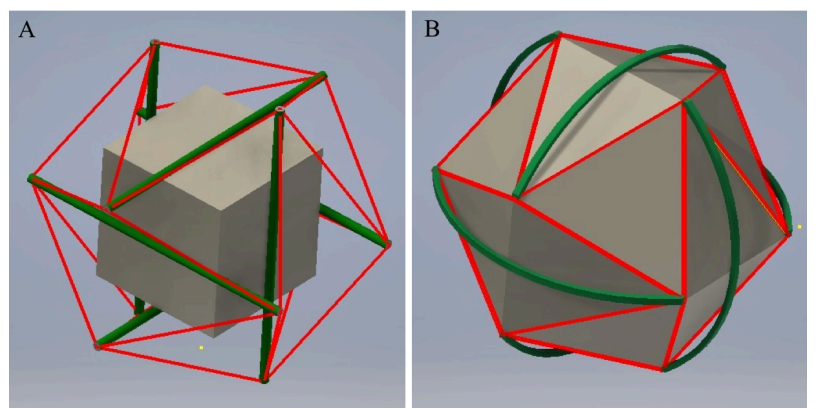

Figure 3. Comparison of the empty volume inside an icosahedron tensegrity cage with straight struts (A) and one with curved struts (B). The empty volume is in grey and is approximated to a cube in the first case and to an icosahedron in the second. The empty volume available with curved struts is 4.7 time larger than the one available with straight struts.

In order to perform repetitive jumps, the propellers must be reoriented upward after each landing (see Section III). Therefore, the core of the robot is designed such that passively self-rights. This behavior is achieved by: 1) decoupling the core of the robot from the cage with a rotation axis housed in two bushes in the tensegrity structure (Fig. 2B); 2) arranging the components of the core such that the overall center of gravity (C.G. in Fig. 2A) is positioned below the axis of rotation. Therefore, the gravity passively reorients upwards the propellers integrated in the core of the robot, hence ensuring repetitive jumps after each landing.

The protective cage is a tensegrity structure [19]. Tensegrity structures are self-stabilized three-dimensional networks composed of two components: the struts, working only in compression and the cables, working only in tension [19]. This feature allows a tensegrity structure to withstand mechanical shocks and be lightweight at the same time [14][19][20]. Both features are beneficial for building a protective cage for jumping robots. Moreover, some tensegrity structures known in literature have an approximated spherical shape that can improve rolling on the ground [21]. Amongst them, the icosahedron tensegrity is the simplest having only 6 struts and 24 cables (Fig. 2B). Furthermore, multiple 
techniques have been proposed for a simple and rapid manufacturing of such tensegrities structures providing a desirable feature for production and deployment of robotic swarms [22][23]. Another useful feature of the tensegrity icosahedron network is the availability of an inner cubic volume not crossed by any cable or strut (Fig. 3A). This volume contains and protects the core of the robot. In this work, the straight struts of the tensegrity icosahedron are replaced by curved struts. This modification increases the internal volume of 4.7 times and facilitates the integration of the two propellers and the whole core without over dimensioning the size and weight of the cage (Fig. 3B). The curved struts also contribute to make the cage more spherical, therefore improving its efficiency for rolling on the ground [24].

\section{LOCOMOTION STRATEGY}

Each element of the proposed mechanical design contributes to a specific step in the locomotion pattern. The aerodynamic damper stabilizes the take-off, the cage allows to roll upon landing, and the rotation axis allows the core to passively up-right before the subsequent take-off. Therefore, thanks to this embodied design, random locomotion can be achieved with a simple control strategy. The controller generates two synchronized duty cycles that periodically give a pulse to the motors that simultaneously activates both the propellers at the maximum thrust. The duration of the pulse has been experimentally optimized in order to maximize the jumping height (see Section IV).

As illustrated in Fig. 4, the simple control strategy leads to two different locomotion patterns: a jumping followed by rolling, or rolling only. When the robot is resting, if the vertical component of the maximum thrust generated by the propellers is larger than the weight of the robot, the propellers generate enough thrust to lift the robot and to initiate a jump
(Fig. 4A). Afterwards, upon landing the robot rolls on the ground. Considering the weight of the robot (37 grams) and the maximum thrust generated by the two propellers (60 grams), this locomotion pattern is obtained when the angle $\alpha$ between the propellers and the gravity is between:

$$
\pm \operatorname{Cos}^{-1}\left(\frac{37}{60}\right)=52^{\circ}
$$

Otherwise, the activation of the propellers results in rolling locomotion only (see Fig. 4B).

\section{IMPLEMENTATION AND CHARACTERIZATION}

The robot is equipped with a TinyDuino board (C) 2017 TinyCircuits) that controls the activation of the propellers. Each motor is driven by a $\mathrm{N}$ channel MOSFFET that is directly controlled by the TinyDuino. For the propulsion, the robot is equipped with two coreless DC motors $(7 \mathrm{~mm}$ in diameter) and two $60 \mathrm{~mm}$ contra-rotating propellers. The robot is equipped with a single cell Lipo battery of $200 \mathrm{mAh}$, that ensures a running time of up to 15 minutes. All these components are connected to a $3 \mathrm{D}$ printed frame that can freely rotate around a single axis of rotation with respect to the cage. The cage is a tensegrity structure composed of a stretched network of cables and curved struts of carbon fiber. The network of cables is $3 \mathrm{D}$ printed flat and subsequently folded and assembled with the struts, as detailed in [22]. The network is printed with NinjaFlex (NinjaTeck, USA), a rubber-like filament that allows to obtain a compliant protective cage to absorb the shocks upon landing.

The robot occupies a spherical volume of $70 \mathrm{~mm}$ of radius and weights 37 grams. Table 1 summarizes the weight of the main components of the robot. Among them, the electronic boards are significantly oversized. Indeed, these components are non-optimized since the simple control algorithm can run on a much simpler, hence lighter board. However, TinyDuino

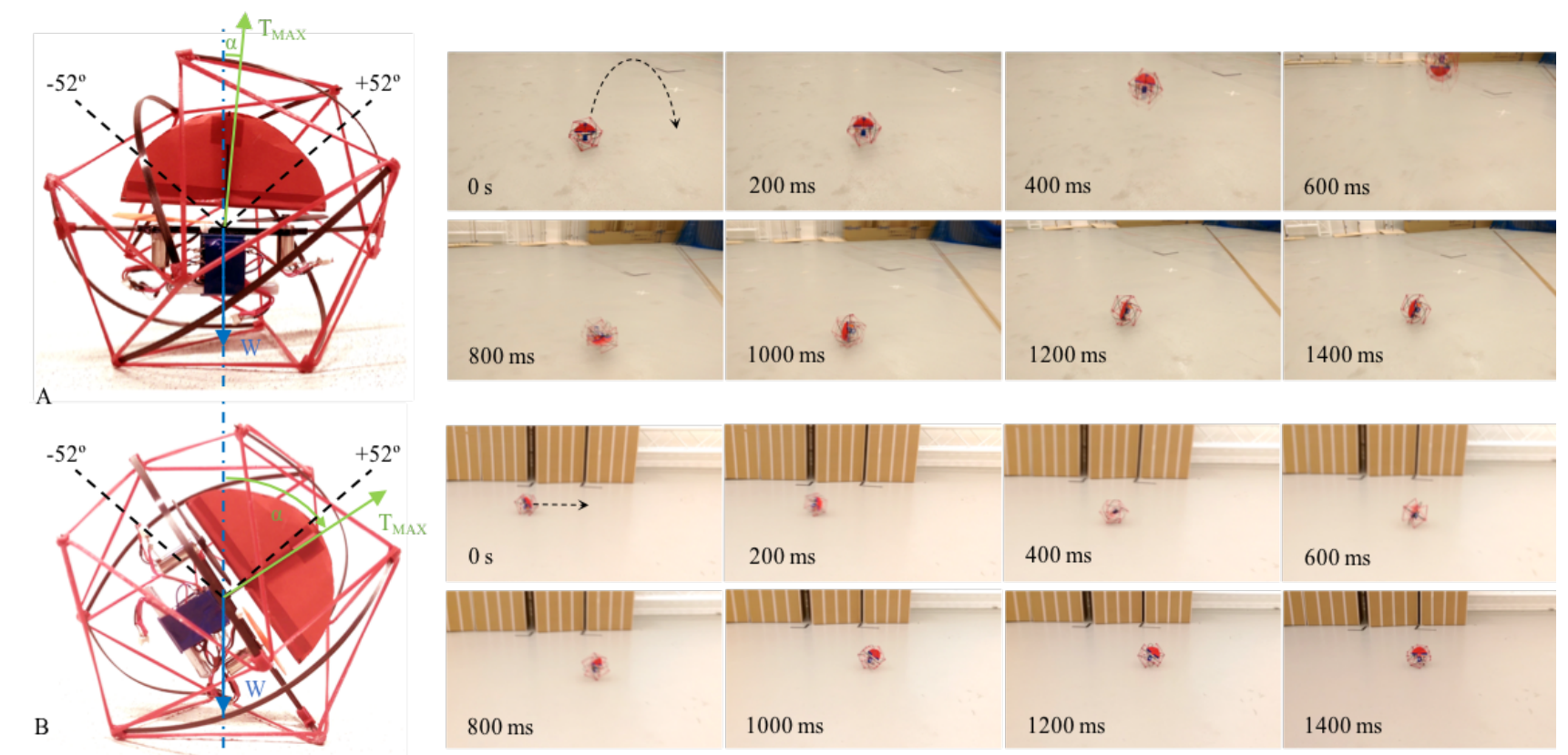

Figure 4. Representative examples of the two locomotion behaviors. (A) If before activation the propellers are within $\pm 52^{\circ}$ from the vertical, the robot jumps first, and subsequently rolls upon landing. (B) Otherwise, the robot rolls without taking-off from the ground. The reader is encouraged to refer to the videos attached to this paper for a better understanding of the locomotion behavior. 
is a convenient choice for prototyping because it allows to thoroughly experiment with the platform by easily tuning different parameters of the control strategy.

Table 1. Weight of the main components of the robot

\begin{tabular}{|c|c|}
\hline Component & Weight \\
\hline Motors and propellers & 10 \\
\hline Core frame & 3 \\
\hline Tensegrity cage & 9 \\
\hline Aerodynamic damper & 1.5 \\
\hline Electronic & 7.5 \\
\hline Battery & 6 \\
\hline
\end{tabular}

The validity of the design has been assessed through three different experimental tests: the height of the jump, the capability to negotiate obstacles, and the amount of area explored by the robot during locomotion.

\section{A. Jumping height}

In order to effectively explore uneven terrains and negotiate obstacles, the robot must be able to perform high jumps. We found that the main parameter affecting the jumping height is the activation time of the propellers. For example, Fig. 5A shows an example of repetitive jumps with a peak of $400 \mathrm{~mm}$ that are obtained when the propellers are activated 1 second every 4 seconds (Fig. 5B).

To find the pulse duration that maximizes the jumping height, the trajectories of multiple jumps for varying activation times have been recorded using an external tracking system, the results are shown in Fig. 5C. The results show that the jumping height gradually increases for activation times up to 1 second, until it reaches a maximum average value of $150 \mathrm{~cm}$. A further increase in the activation time does not result in an increasing jumping height. Most probably because the passive stabilization offered by the aerodynamic damper is effective only in the first phase of the jump and loses efficacy over 1 second. Increasing the time of propeller activation only results in landing with the propellers still active, hence high impact velocities that increase the risk of damaging the robot. For the next experiments, an activation time of 1 second and a duty cycle with a period of 4 seconds have been selected.

\section{B. Obstacle negotiation}

The purpose of the experiment is to investigate the mobility of the robot in uneven terrains by measuring how quickly the robot can escape from a box of $50 \times 50 \mathrm{~cm}$ with walls of different height. For each height of the walls, the number of duty cycles, and time required to exit the box have been logged. The results are summarized in Table 2 and in Fig. 6.
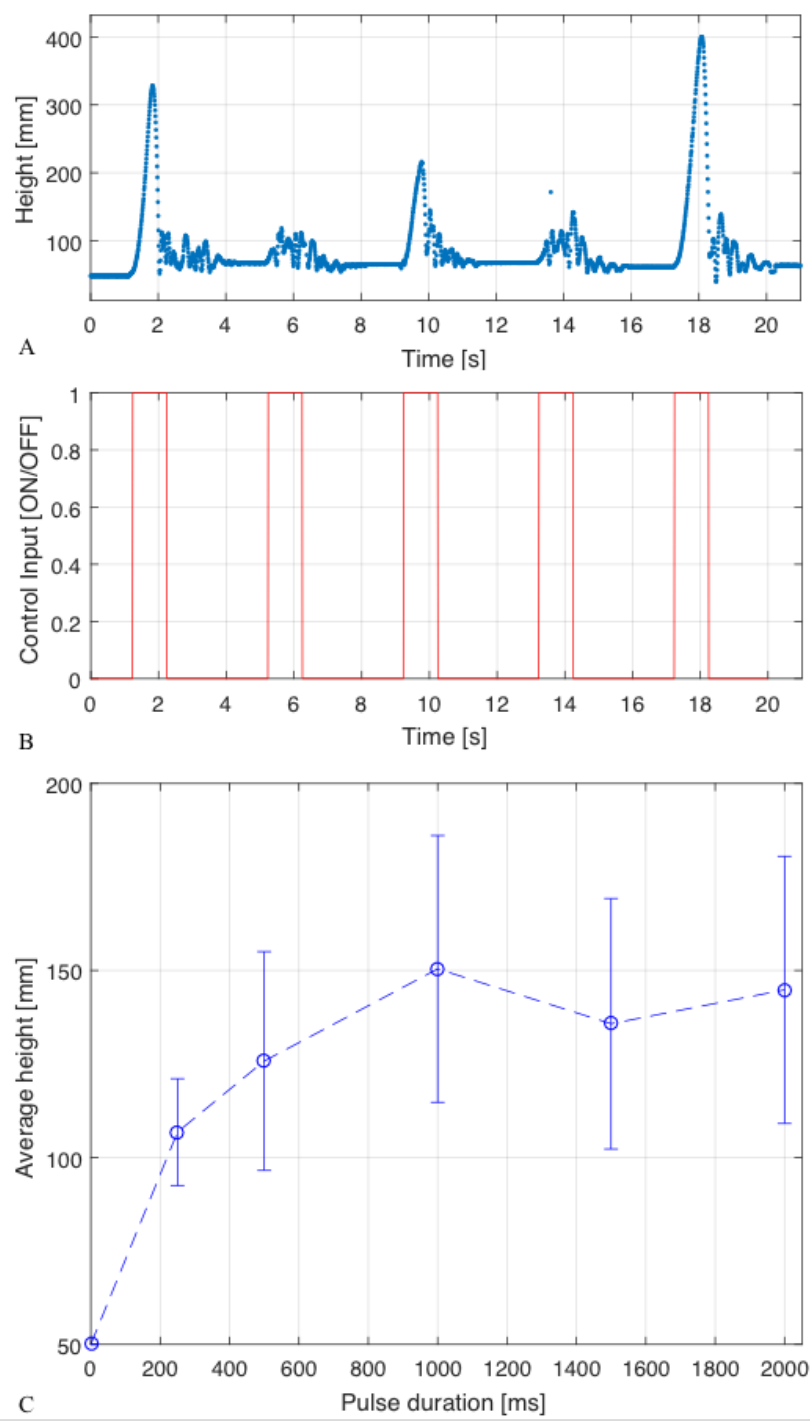

Figure 5. (A) Example of jumping trajectoris with the aforementioned control input. (B) Example of commands to the propllers with activation time of 1.5 seconds and period of 4 secons. (C) Average jumping height in function of different pulse durations $(250,500,1000,1500$ and $2000 \mathrm{~ms})$

Until a height of $60 \mathrm{~mm}$, the robot escaped from the box in less than 10 seconds and with maximum 3 iterations. For walls $70 \mathrm{~mm}$ high, the robot managed to jump over the walls with an average of 5.5 duty cycles in less than 40 seconds. Above $70 \mathrm{~mm}$, the robot takes considerable time to exit the box. This experiment suggest that the current prototype can rapidly search environments containing obstacles with a maximum height of $70 \mathrm{~mm}$, but the exploration will significantly slow-down in environments with higher obstacles.

Table 2. Summary of the number of iterations and time required by the prototype to exit from a $50 \times 50 \mathrm{~cm}$ box with different wall height

\begin{tabular}{|c|c|c|c|c|c|c|c|c|c|c|c|}
\hline \multicolumn{2}{|c|}{$50 \mathrm{~mm}$} & \multicolumn{2}{c|}{$60 \mathrm{~mm}$} & \multicolumn{2}{c|}{$70 \mathrm{~mm}$} & \multicolumn{2}{c|}{$80 \mathrm{~mm}$} & \multicolumn{2}{c|}{$90 \mathrm{~mm}$} & \multicolumn{2}{c|}{$100 \mathrm{~mm}$} \\
\hline $\begin{array}{c}\text { \# of } \\
\text { jumps }\end{array}$ & Time (s) & $\begin{array}{c}\text { \# of } \\
\text { jumps }\end{array}$ & Time (s) & $\begin{array}{c}\text { \# of } \\
\text { jumps }\end{array}$ & Time (s) & $\begin{array}{c}\text { \# of } \\
\text { jumps }\end{array}$ & Time (s) & $\begin{array}{c}\text { \# of } \\
\text { jumps }\end{array}$ & Time (s) & $\begin{array}{c}\text { \# of } \\
\text { jumps }\end{array}$ & \begin{tabular}{c} 
Time (s) \\
\hline 3
\end{tabular} \\
\hline 3.4 & 1 & 0.7 & 3 & 11.3 & 9 & 38.3 & 41 & 118 & $\mathrm{x}$ & $\mathrm{x}$ \\
\hline 1 & 0.8 & 1 & 0.6 & 3 & 9.5 & 23 & 66 & 4 & 10 & 2 & 59 \\
\hline 1 & 0.8 & 2 & 4.7 & 9 & 3.4 & 5 & 11 & $\mathrm{x}$ & $\mathrm{x}$ & $\mathrm{x}$ & $\mathrm{x}$ \\
\hline 1 & 0.5 & 2 & 5.7 & 3 & 8.1 & 39 & 118 & $\mathrm{x}$ & $\mathrm{x}$ & 6 & 21 \\
\hline 2 & 4.2 & 3 & 9.8 & 9 & 38.6 & $\mathrm{x}$ & $\mathrm{x}$ & 11 & 42 & $\mathrm{x}$ & $\mathrm{x}$ \\
\hline
\end{tabular}




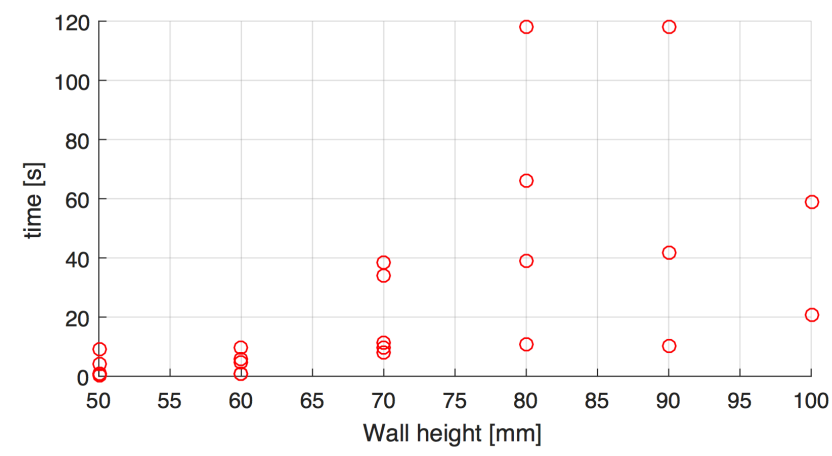

Figure 6. The graph shows the time required by the robot to exit from a $50 \times 50 \mathrm{~cm}$ box with walls with different wall heights.

\section{Area coverage}

To assess the area coverage capability of the robot, we tracked the path of the robot during the random exploration of a flat arena of $10 \times 10 \mathrm{~m}$. The top view of the trajectories resulting from 9 runs are shown in Fig. 7A. Each run lasted 2 minutes (corresponding to roughly 30 jumps). The starting point of the robot was at the origin $(0,0)$ of the arena. The robot was oriented with the two propellers aligned along the $\mathrm{Y}$ axis. In order to evaluate the effectiveness of the exploration, the arena has been divided into cells of the same size of the robot $(140 \times 140 \mathrm{~mm})$. The effectiveness of coverage was then measured as the ratio between the corresponding area of cells that were visited at least once and the total area of the arena [25]. As shown in Fig. 7B, a single robot can explore at an almost constant average rate of 0.044 $\mathrm{m}^{2} / \mathrm{s}$. We can also use this experiment to estimate how effectively a swarm composed of 9 agents that starts from the same location can explore an area of interest. Fig. 7C shows the area that the swarm can explore as function of the time. The swarm has an almost constant exploration rate of 0.27 $\mathrm{m}^{2} / \mathrm{s}$, corresponding to $16 \mathrm{~m}^{2}$ explored in just one minute. Fig. $7 \mathrm{D}$ shows the percentage of surface covered in 2 minutes by the swarm in a circular region centered in the origin where the robots are deployed. The percentage of explored area is decreasing almost linearly with the radius of the region.

\section{DISCUSSION}

The exploration of unknown environments is an active field of research in robotics. One solution is to systematically explore the environment by carefully planning the path of a robot. This approach requires robots with mechanisms and electronics suited for a highly controllable locomotion [13][14]. Another solution is to explore the environment with many simple robots capable of random locomotion. This exploration strategy is preferred when the information regarding positional or behavioral characteristics of targets decreases [6]. In this paper, we have presented a new soft robot for the random exploration of unstructured terrestrial environments. The robot has an embodied design where each mechanical component directly contributes to the jumping and rolling locomotion without the need of complex control algorithms. The design solutions exploited for the jumping mechanism, self-righting mechanism and protective tensegrity cage have been selected for their intrinsic mechanical simplicity and ease of manufacture that could facilitate the development of several agents for swarm applications.
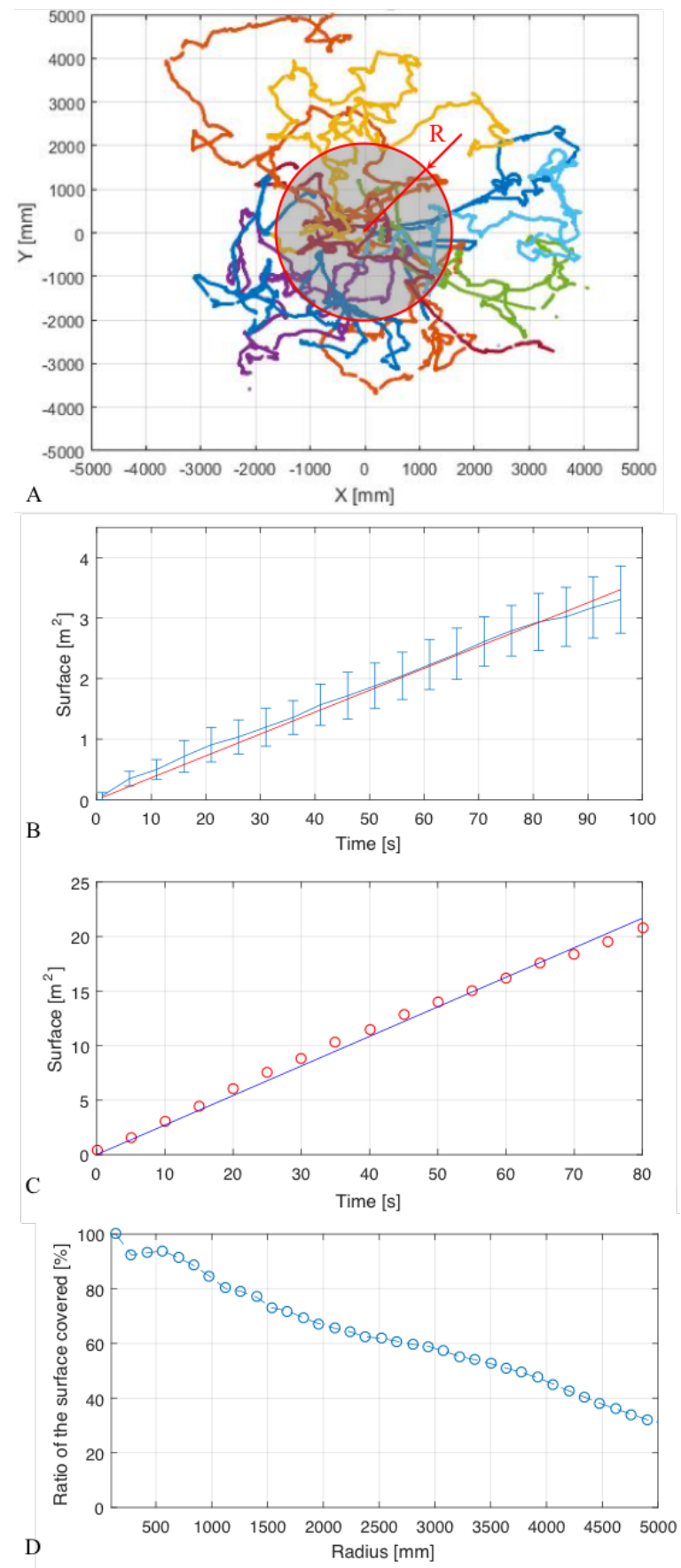

Figure 7. (A) Trajectories resulting from 9 tests of the random explorer. (B) Average area covered by a single random explorer as function of time. (C) Area that a swarm of 9 robots can cover as function of time. (D) Percentage of surface covered in 2 minutes by the swarm in the circular region centered as function of its radius.

The current prototype demonstrated the capability to rapidly explore flat and uneven terrains with small obstacles like pebbles, as shown in the supplementary video material. However, the limited average jumping height affects the 
exploration rate of the robot when deployed in terrains with obstacles higher than $70 \mathrm{~mm}$. Increasing the thrust to weight ratio of the robot is a viable approach to tackle this limitation. For example, by replacing the two DC motors with brushless motors (e.g. DYS BE0905), the thrust could increase by $80 \%$. The thrust to weight ratio can be also increased by rearranging the motors in a coaxial configuration. This would improve the propulsion efficiency and reduce the footprint of the robot, therefore the weight of the cage. Another option for jumping higher would be to increase the area of the aerodynamic dampers in order to improve stabilization during the take-off phase beyond the 1 second currently obtained.

A lighter electronic board with only the few components required to periodically activate the motors would allow to allocate payload to sensors and communication devices to collect and to stream data to the user.

Finally, the use of several units for swarm operation would require tackling the challenge of deployment, for example with techniques of self-folding for tensegrity structures to facilitate the storage and transportation of the robots [26]. This would result in a highly portable and lightweight swarm that can be easily carried inside a backpack and rapidly deployed on the field.

\section{ACKNOWLEDGMENT}

This work was supported by the Swiss National Science Foundation through the National Centre of Competence in Research Robotics (NCCR Robotics).

\section{REFERENCES}

[1] R. R. Murphy, S. Tadokoro, D. N. Jacoff, P. Fiorini, H. Choset, and Aydan M Erkmen, "Search and Rescue Robotics," in Springer Handbook of Robotics, 2008, pp. 1151-1173.

[2] S. Dubowsky, K. Iagnemma, S. Liberatore, D. M. Lambeth, J. S. Plante, and P. J. Boston, "A Concept Mission: Microbots for LargeScale Planetary Surface and Subsurface Exploration," in Intl. Forum for Space Technology and Applications, M. S. El-Genk and M. J. Bragg, Eds., vol. 746, no. 1. Albuquerque, NM: AIP, Feb. 2005, pp. 1449-1458.

[3] P. S. Schenker, T. L. Huntsberger, P. Pirjanian, E. T. Baumgartner, and E. Tunstel, "Planetary Rover Developments Supporting Mars Exploration, Sample Return and Future Human-Robotic Colonization," Auton. Robots, vol. 14, no. 2, pp. 103-126, 2003.

[4] M. Dunbabin and L. Marques, "Robots for Environmental Monitoring: Significant Advancements and Applications," IEEE Robotics \& Automation Magazine, vol. 19, no. 1. pp. 24-39, 2012.

[5] D. Goense, E. J. van Henten, and C. Lokhorst, "Precision agriculture '09." Wageningen Academic Publishers, p. 992, 01-Jul-2009.

[6] V. Méndez, D. Campos, F. Bartumeus, Random search strategies, in: Stochastic foundations in movement ecology, Springer, 2014, pp. 177-205.

[7] W.H.Oskay,"Bristlebot: A tiny directional vibrobot,"2007.[Online]. Available: http://www.evilmadscientist.com/article.php/bristlebot

[8] Y. Han, H. Marvi, and M. Sitti, "Fiberbot: A miniature crawling robot using a directional fibrillar pad," Robot. Autom. (ICRA), 2015 IEEE Int. Conf., pp. 3122-3127, 2015.

[9] G. Bonsignori, C. Stefanini, U. Scarfogliero, S. Mintchev, G. Benelli, and P. Dario, "The green leafhopper, Cicadella viridis (Hemiptera, Auchenorrhyncha, Cicadellidae), jumps with near-constant acceleration.," J. Exp. Biol., vol. 216, no. Pt 7, pp. 1270-9, 2013.

[10] M. Kovač, M. Schlegel, J. C. Zufferey, and D. Floreano, "Steerable miniature jumping robot," Auton. Robots, vol. 28, no. 3, pp. 295-306, 2010.
[11] J. Burdick and P. Fiorini, "Minimalist Jumping Robots for Celestial Exploration,” Int. J. Rob. Res., vol. 22, no. 7-8, pp. 653-674, 2003.

[12] D. W. Haldane, M. M. Plecnik, J. K. Yim, and R. S. Fearing, "Robotic vertical jumping agility via series-elastic power modulation," Sci. Robot., vol. 1, no. 1, Nov. 2016.

[13] B. J. Hockman, A. Frick, R. G. Reid, I. A. D. Nesnas, and M. Pavone, "Design, Control, and Experimentation of Internally-Actuated Rovers for the Exploration of Low-gravity Planetary Bodies," J. F. Robot., vol. 34, no. 1, pp. 5-24, 2017.

[14] K. Kim, L. H. Chen, B. Cera, M. Daly, E. Zhu, J. Despois, A. K. Agogino, V. Sunspiral, and A. M. Agogino, "Hopping and rolling locomotion with spherical tensegrity robots," in IEEE International Conference on Intelligent Robots and Systems, 2016, vol. 2016November, pp. 4369-4376.

[15] Y. Sugiyama and S. Hirai, "Crawling and Jumping by a Deformable Robot,” Int. J. Rob. Res., vol. 25, no. 5-6, pp. 603-620, May 2006.

[16] S. Li, R. Katzschmann, and D. Rus, "A soft cube capable of controllable continuous jumping," Intelligent Robots and Systems (IROS), 2015 IEEE/RSJ International Conference on. pp. 1712-1717, 2015.

[17] N. W. Bartlett, M. T. Tolley, J. T. B. Overvelde, J. C. Weaver, B. Mosadegh, K. Bertoldi, G. M. Whitesides, and R. J. Wood, "A 3Dprinted, functionally graded soft robot powered by combustion," Science (80-. )., vol. 349, no. 6244, p. 161 LP-165, Jul. 2015.

[18] Z. E. Teoh, S. B. Fuller, P. Chirarattananon, N. O. Prez-Arancibia, J. D. Greenberg, and R. J. Wood, "A hovering flapping-wing microrobot with altitude control and passive upright stability," in IEEE International Conference on Intelligent Robots and Systems, 2012, pp. 3209-3216.

[19] R. E. Skelton and M. C. de Oliveira, Tensegrity systems. New York, NY, USA: Springer Science+Business Media, ch. 1.

[20] L.-H. Chen, K. Kim, E. Tang, K. Li, R. House, E. L. Zhu, K. Fountain, A. M. Agogino, A. Agogino, V. Sunspiral, and E. Jung, "Soft Spherical Tensegrity Robot Design Using Rod-Centered Actuation and Control," J. Mech. Robot., vol. 9, no. 2, p. 25001, 2017.

[21] A. Agogino, V. SunSpiral and D. Atkinson, "SuperBall Bot Structure for planetary landing and exploration" Final Report for the NASA Innovative Advanced Concepts, NASA Ames Reasearch Center, Intelligent Systems Division, Jul. 2013.

[22] D. Zappetti, S. Mintchev, J. Shintake, and D. Floreano, "Bio-inspired Tensegrity Soft Modular Robots BT - Biomimetic and Biohybrid Systems: 6th International Conference, Living Machines 2017, Stanford, CA, USA, July 26-28, 2017, Proceedings," M. Mangan, M. Cutkosky, A. Mura, P. F. M. J. Verschure, T. Prescott, and N. Lepora, Eds. Cham: Springer International Publishing, 2017, pp. 497-508.

[23] L. Chen, et al., "Modular elastic lattice platform for rapid prototyping of tensegrity robots", Proceedings of the ASME 2017 International Design Engineering Technical Conferences \& Computers and Information in Engineering Conference.

[24] T. Kaufhold, F. Schale, V. Böhm, and K. Zimmermann, "Indoor locomotion experiments of a spherical mobile robot based on a tensegrity structure with curved compressed members," in 2017 IEEE International Conference on Advanced Intelligent Mechatronics (AIM), 2017, pp. 523-528.

[25] S. C. Wong, L. Middleton, and B. a Macdonald, "Performance metrics for robot coverage tasks," in In Proceedings Australasian Conference on Robotics and Automation (ACRA, 2002, no. November, pp. 7-12.

[26] T. Liedl, B. Hogberg, J. Tytell, D. E. Ingber, and W. M. Shih, "Selfassembly of three-dimensional prestressed tensegrity structures from DNA," Nat Nano, vol. 5, no. 7, pp. 520-524, Jul. 2010. 